\title{
Geography, Health, and the Pace of Demo-Economic Development
}

\author{
Holger Strulik ${ }^{*}$ \\ Leibniz Universität Hannover, Discussion Paper No. 361
}

ISSN 0949-9962

April 2007

\begin{abstract}
This paper investigates the impact of subsistence consumption and extrinsic and intrinsic causes of child mortality on fertility and child expenditure. It offers a theory for why mankind multiplies at higher rates at geographically unfavorable, tropical locations. Placed into a macroeconomic framework this behavior creates an indirect channel through which geography shapes economic performance. It is explained why it are countries of low absolute latitude where we observe exceedingly slow (if not stalled) economic development and demographic transition.
\end{abstract}

Keywords: Demographic Transition, Geography, Health, Nutrition, CrossCountry Divergence

JEL: J10, J13, O11, O12

\footnotetext{
*Department of Economics, University of Hannover, Koenigsworther Platz 1, 30167 Hannover, Germany. Email: strulik@vwl.uni-hannover.de. I would like to thank Carl-Johan Dalgaard, Oded Galor, Volker Grossmann, Omer Moav, Fidel Perez Sebastian, Nikolaus Siegfried, participants at seminars in Copenhagen, Hannover, and Vienna, and three anonymous referees for useful comments.
} 


\section{INTRODUCTION}

As for every species, survival of humans is easier in some regions of the world and harder in others. In particular tropical regions - defined by an absolute latitude below $23.5^{\circ}$ - provide an unfavorable location for a child to survive whereas survival is almost certain at latitudes of $40^{\circ}$ and higher. This fact is visualized in Figure 1.a which shows for 137 countries average absolute latitude against the probability for a child to survive its fifth birthday. ${ }^{1}$

Figure 1: Absolute Latitude against Child Survival (left) and Population Growth (right)
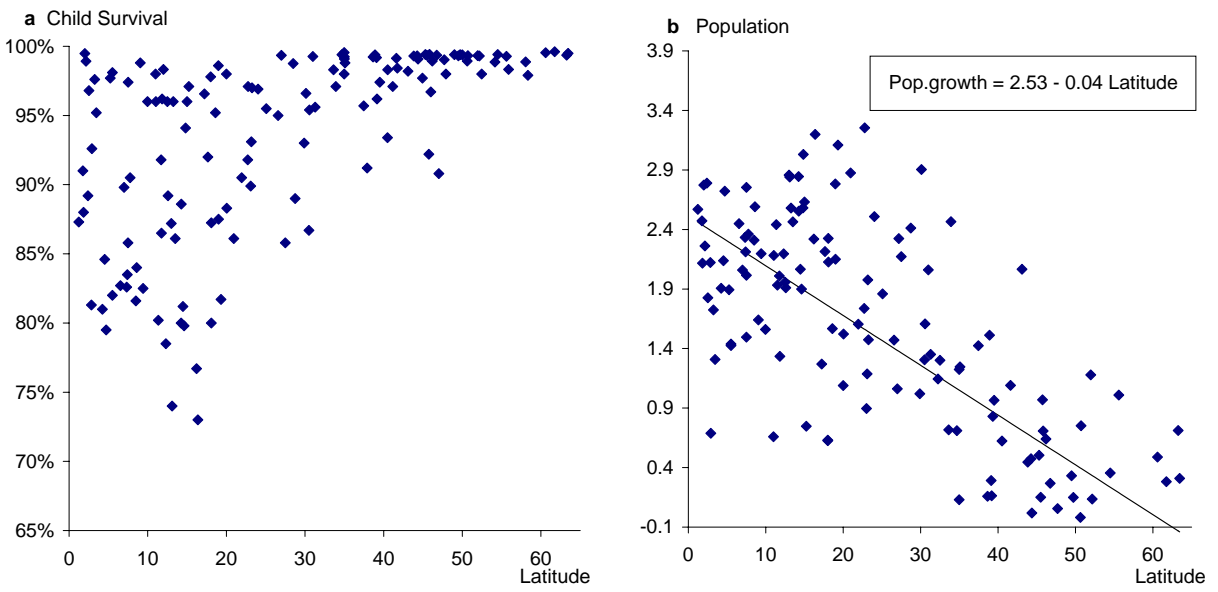

a) Child Survival Rate=1- Under-5-Mortality Rate, Year 2000, b) $R^{2}=0.506$. Data from World Bank (2004) and Masters and McMillan (2001).

Interestingly, the human population grows at higher rates at low geographic latitudes. This striking fact is shown in Figure 1.b. From a biological viewpoint this behavior of humans seems bizarre. Why should a species multiply at higher rates in environments for which it is less fit to live in?

A seemingly obvious explanation is the demographic transition according to which fertility follows a decline of mortality with delay so that the time path of population growth describes an inverted $\mathrm{u}$, rising in its initial phase and falling later. Given that the demographic transition started earlier at geographically favorable locations, a negative correlation between latitude and population growth follows automatically. High-latitude countries have accomplished the transition already, resting at low mortality and fertility rates. Low-latitude countries have

\footnotetext{
${ }^{1}$ We focus on child survival rates, which will be the crucial variable in the theoretical model. Similar figures can be drawn for infant survival and longevity. As Schultz (1999) notes, intercountry differences in life expectancy are dominated by rates of infant and child survival.
} 
experienced some reduction of mortality but this has not yet triggered a comparable decline of fertility rates. The problem with this argument is that a picture similar to Figure 1.b could have been drawn for every decade for which the data are available. A more appropriate description of the empirical regularities is thus that the population in geographically unfavorable regions grows at higher rates along the invertedly u-shaped transition path.

A study by Reher (2004) supports this view. It classifies the world's countries according to their position in the demographic transition into forerunners, followers, trailers, and latecomers. A central result is that population growth along the transition path peaks at the lowest rate for forerunners, at considerably higher rates for followers and trailers, and at the highest rate for latecomers. The group of forerunners contains almost exclusively European and Northern American countries located at high latitudes with temperate climate. Followers and trailers (mostly Asian and South American countries) are less favorably located, on average just inside the tropics, while latecomers (mostly from Sub-Saharan Africa) are clearly tropically located. The observation of regional-specific patterns of demographic transition is confirmed in Figure 2. It shows the historical peak of population growth - assumed at country specific years between 1870 (in Sweden) and 1990 (in Nigeria) - against absolute latitude for 128 countries. Population growth tends to be higher along the path of demographic transition for countries of lower absolute latitude.

Figure 2: Absolute Latitude and Historical Peak of Population Growth for 128 Countries

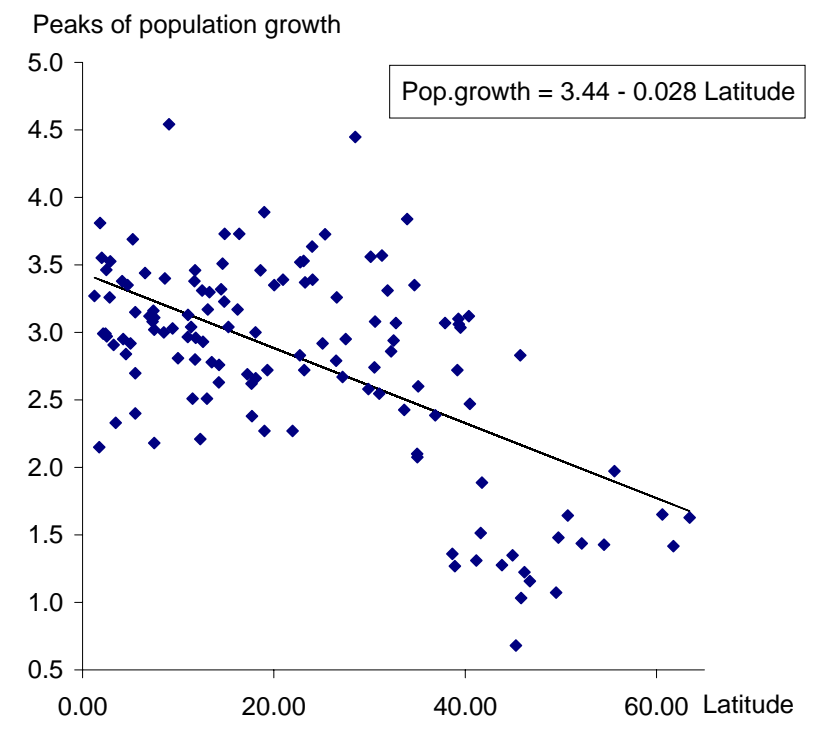

Data from Reher (2004) and Masters and McMillan (2001). 
This paper proposes a novel theory that explains not only the inverted u-pattern of population growth but also why the inverted u-shaped curve shifts upwards (i.e. in direction of higher population growth rates ) when absolute latitude decreases. Placed into a macroeconomic context this mechanism can explain why economic and demographic development is slower at tropical locations and why tropically located countries are particularly prone to get stuck in a poverty trap.

A key element of the theory is a partition of child survival rates into extrinsic and intrinsic components. While the extrinsic part is exogenous to the individual parent the intrinsic part is individually controllable through expenditure on child nutrition and health. The wording was inspired by an analogy to extrinsic and intrinsic mortality in evolutionary biology and disposablesoma theory (Williams, 1957, Kirkwood and Austad, 2000). Yet, although the analogy to biology is visible, it is far from perfect. We focus on child mortality and treat individuals (i.e. parents) as optimizing agents whereas biology is concerned with senescence and nature (through evolution) is the optimizing "agent". Consider, for example, a mouse whose main extrinsic cause of death is cold. According to the disposable-soma theory the mouse benefits by "investing" spare resources into thermogenesis or reproduction rather than into better cell repair. It is thus the interplay between extrinsic mortality (through ambient temperature) and intrinsic mortality (through optimizing nature) that explains the short life-span and high fertility rates of mice.

In this paper, the intrinsic part of child survival depends on nutrition, i.e. the part of parental income devoted to child expenditure. Nutrition affects the weight and height of children and a child's height-for-age and weight-for-height are commonly used as predictors of morbidity and mortality (Dasgupta, 1993, Ch. 4). Besides the direct effects on starvation there exists a probably more important yet frequently overlooked indirect channel, which is nutrition's interaction with diseases. In its year 2002 Report the World Health Organization listed child underweight as the leading risk factor contributing to the global burden of disease. Most studies agree in their assessment that about $55 \%$ of the annual 10 Million deaths of children younger than 5 years can be attributed to undernutrition ranging from $45 \%$ for deaths because of measles to $61 \%$ for deaths because of diarrhoea. ${ }^{2}$

The notion that most deaths could have been avoided if more money were spent on nutrition and health care accords with cross-country studies provided in the economics literature. These

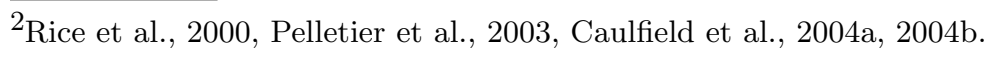


usually find a strong correlation of child mortality or, more generally, life expectancy and health with income per capita and causality running from income to health, see Pritchett and Summers, 1996, and the literature cited therein. ${ }^{3}$

While some authors emphasize a predominantly indirect effect of geographic location and disease environment on economic performance through institution building at colonoial times (Acemoglu et al., 2001) others argue in favor of an independent influence of geography that makes some regions inherently less healthy than others (Bloom and Sachs, 1998, Schultz, 1999, Sachs, 2003, and Olsson and Hibbs (2005). For example, falciporom malaria needs an ambient temperature of $22^{\circ} \mathrm{C}$ or above during the incubation period. Generally, biologists find that geographic latitude is a good predictor of disease species diversity with the highest diversity observed in tropical regions (Brown, 1995). Another explanation besides temperature's direct effects on metabolism is that winter frost eliminates the prevalence of many pathogens and parasites (Masters and McMillan, 2001).

For the demo-economic model proposed in this paper we will thus assume that intrinsic child survival rates are determined by individual child expenditure and extrinsic child survival rates are determined by the absolute geographic latitude and the state of economic development of a family's country or region of residence. The state of economic development is here summarized by average income per capita and subsumes factors like, for example, (income tax-financed) public health spending.

In order to explain the impact of geography on the demographic transition it would not be sufficient to explain why parents facing higher extrinsic child survival rates adjust with higher fertility rates. ${ }^{4}$ We need to explain a superproportional reaction according to which they adjust with higher net rates of reproduction. It will be shown that this human behavior can be provoked by a particular form of decreasing returns on child expenditure: An additional fraction of income spent on children is relatively ineffective in preventing death when the family lives in

\footnotetext{
${ }^{3}$ With contrast to the medical literature, which continuously emphasizes the impact of nutrition on child mortality, analyses by economists and demographers appear to be somewhat less consistent. The survey article by Cutler et al. (2006) "downplays" the causal mechanism running from income to health and emphasizes scientific advance and public health as determinants of mortality. The cross-country study by Filmer and Pritchett (1999), however, finds only a numerically small and statistically insignificant impact of public health spending on child mortality. Harris (2004) provides a recent re-assessment and qualified confirmation of the McKeown's (1976) thesis about the dominant role of nutrition in the decline of mortality during England's demographic transition. See also Fogel (1994, 2004).

${ }^{4}$ This result would be compatible with simple economic reasoning as well as with life-cycle theory of biology. It is confirmed across countries by Guegan et al. (2001) who find - controlling for various economic social and demographic patterns - a strong positive correlation between fertility rates and diversity of disease species.
} 
a rich country at high geographic latitude where child survival rates are high anyway. On the other hand, it can "buy" a large improvement of survival chances when the family lives in an environment where extrinsic child survival rates are low. This immediately intuitive mechanism will be called the health multiplier. ${ }^{5}$

For a preliminary intuition of why the health multiplier can generate the observed fertility pattern consider an exogenous increase of the extrinsic child survival rate. To this parents adjust with having less children not only because more children survive anyway but also because their individual income becomes less effective in preventing child death i.e. because the shadow price of a surviving child rises; an additional effect that makes the wish for a large family unattractive. This "double causation" induces a superproportinal reaction with lower fertility. Ceteris paribus, parents in low mortality environments prefer small families whereas parents in high mortality environments prefer large ones.

The interplay of the health multiplier with a hierarchy-of-needs effect resulting from subsistence requirements generates an invertedly u-shaped correlation between income and population growth. This result is derived in Section 2 (which sets up the model) and Section 3 (which obtains the implied correlations). Section 4 integrates the theory into a macroeconomic framework. Section 5 explores how geographic location affects the possibility of economic stagnation. Section 6 investigates the impact of geography on the pace of demo-economic development using numerical calibrations of the model. Related literature is discussed in Section 7. Section 8 concludes.

\section{The Model}

Consider the decision problem of a parent who derives utility from consumption $(c)$, from having a family ( $\tilde{n}$ children), and from child expenditure $(h)$. Abstracting from gender differences any adult is allowed to reproduce without matching. Actual family size, $\tilde{n} \equiv n \cdot \pi$, differs from fertility, $n$, because the child survival rate is less than one. To keep the analysis tractable we consider $n$ as continuous variable. Thus, the parent under investigation can be regarded as an economy's average adult who bears $n$ children, spends a fraction $h$ of his income on each child, and observes a fraction $\pi$ of them surviving childhood. Survival during adulthood is assumed

\footnotetext{
${ }^{5}$ See Strulik (2005) for empirical support of the health multiplier.
} 
to be certain. ${ }^{6}$ Summarizing, his or her utility is given by

$$
u(c, \tilde{n}, h)=\log (c-\bar{c})+\beta_{1} \log (n \cdot \pi(\bar{\pi}, h))+\beta_{2} \log (h)
$$

if $c>\bar{c}$. Otherwise, $c=y$ (and $u=-\infty)$.

In order to obtain a closed form solution the utility function is logarithmic. Because of the incidence of subsistence consumption, $\bar{c}$, this assumption is less restrictive than usually. The intertemporal elasticity of substitution is - in deviation to standard models - not constant during the process of economic development. It assumes a value of zero at subsistence level and converges towards one as income and consumption go to infinity.

The incidence of subsistence consumption generates a hierarchy of needs. If income raises from subsistence level, current consumption becomes less important and young adults increasingly care about the size of their family. The positive income effect on fertility is largest close to subsistence level and vanishes as income goes to infinity and $\bar{c}$ becomes negligible small relative to income.

Child expenditure $h$ plays a double role in the model. The $\beta_{1}$ part of utility takes into account that parents - observing extrinsic survival probabilities $\bar{\pi}$ for their children - can influence survival through child expenditure. Additionally, parents are allowed to derive utility from child expenditure directly. This utility, captured by the $\beta_{2}$ component, could originate either from a "warm glow of giving" (Andreoni, 1989) or a preference for having higher quality children (Becker, 1960). It helps to explain why parents increase child expenditure with economic development although additional units of child expenditure have a decreasing and eventually vanishing effect on child survival. Thus, the variable $h$ can be thought of human capital in a broad sense, consisting mainly of health and nutrition when income is low and of schooling expenditure at high incomes. ${ }^{7}$

As motivated in the Introduction, child survival is understood as a complement consisting of extrinsic and intrinsic parts. The extrinsic part $\bar{\pi}$ is exogenous to the individual parent and determined by geographic location $g$ and average income per capita produced in the economy $\bar{y}$. The intrinsic part of child survival depends positively on the fraction of income spent on child

\footnotetext{
${ }^{6}$ See Strulik (2006) for an extension of the model with a third period and savings financed old-age consumption. ${ }^{7}$ It would be possible to separate these effects by introducing different choice variables for the nutrition and schooling components of human capital. This, however, would mainly add notational clutter and produce little value added. Note that alternatively $h$ could be interpreted as the time spent on child-rearing per child.
} 
nutrition and health care. Here we assume a particular form of decreasing returns. An additional unit of child expenditure is less effective in preventing death at higher extrinsic survival rates. In order to clarify the role of this mechanism we introduce the parameter $\mu$, which will be called the health multiplier. Summarizing, child survival is given by

$$
\begin{array}{ll}
\pi=\bar{\pi}+[1-\mu \cdot \bar{\pi}] \cdot \lambda \cdot h \quad & \mu \geq 0, \lambda>0 . \\
\bar{\pi}=\bar{\pi}(\bar{y}, g), \quad \frac{\partial \bar{\pi}}{\partial \bar{y}}>0, & \frac{\partial \bar{\pi}}{\partial g}>0, \quad \lim _{\bar{y} \rightarrow \infty} \frac{\partial \bar{\pi}}{\partial \bar{y}}=0, \quad \lim _{\bar{y} \rightarrow \infty} \bar{\pi}=a<1,
\end{array}
$$

where $\lambda$ denotes general productivity of child expenditure. In accordance with the empirical evidence income and absolute latitude exert a positive effect on child survival and the marginal income effect on child survival vanishes as income goes to infinity.

Given that the health multiplier operates $(\mu>0)$, the return on health expenditure $\partial \pi / \partial h=$ $[1-\mu \cdot \bar{\pi}] \cdot \lambda$ is decreasing in extrinsic survival $\bar{\pi}$. From a marginal cost viewpoint parents located in an environment of low extrinsic survival rates have an advantage in producing children whereas parents in an environment of anyway high survival rates $\bar{\pi}$ face less effective child expenditure with respect to child survival and thus family size. Consequently, when $\bar{\pi}$ is low, child expenditure will be mainly motivated by the desire to have surviving offsprings. It will therefore consist to a large extent of nutrition and health care expenditure (the $\beta_{1}$ part). On the other hand, at high $\bar{\pi}$ 's, expenditure will be mostly motivated by its education component (the $\beta_{2}$ part). Occasionally, we will set the health multiplier to zero to emphasize this effect.

Adults supply one unit of labor and receive labor income $y$ implying the budget constraint

$$
y=c+n h y .
$$

Maximization of (1) subject to (2) and (4) provides the following first order conditions for an interior solution.

$$
\begin{aligned}
& \frac{\partial u}{\partial \tilde{n}} \pi=\frac{\partial u}{\partial c} h y \quad \Rightarrow \quad \frac{\beta_{1}}{n}=\frac{1}{c-\bar{c}} h y, \\
& \frac{\partial u}{\partial \tilde{n}} n \frac{\partial \pi}{\partial h}+\frac{\partial u}{\partial h}=\frac{\partial u}{\partial c} n y \quad \Rightarrow \quad \frac{\beta_{1}}{\pi}(1-\mu \bar{\pi}) \lambda+\frac{\beta_{2}}{h}=\frac{1}{c-\bar{c}} n y .
\end{aligned}
$$

Condition (5a) requires that utility from having another child equates child costs in terms of foregone utility from consumption. According to condition (5b) utility from spending an additional unit of income on children - derived indirectly through the impact of health expenditure 
on family size and directly through higher quality children (or the warm glow of giving) - equates marginal child costs in terms of foregone utility from consumption. Holding consumption constant we see from (5a) that higher child expenditure $(h \cdot y)$ is observed together with the desire for a smaller family (lower $n$ and higher $\partial u / \partial \tilde{n}$ ). This is the Beckerian child quantity-quality trade-off.

The presence of mortality and health expenditure generates a second quantity-quality tradeoff visible in (5b). To understand its consequence consider an increase of extrinsic survival probability $\bar{\pi}$. This has a twofold negative effect on the first term on the left hand side: First, marginal utility from health expenditure driven by the wish for a large family $\left(\partial u / \partial \tilde{n} \cdot n=\beta_{1} / \pi\right)$ decreases, because more children survive anyway. Second, marginal returns of health expenditure are lower because child expenditure is less effective in preventing death under the generally improved survival conditions. As a consequence parents reduce their fertility rate and the right hand side of $(5 b)$ decreases.

Now, with decreasing fertility, the familiar Beckerian trade-off in (5a) becomes operative and parents want to spend more on their children. The increase of $h$ has a positive feedback effect on the left-hand side of $(5 \mathrm{~b})$ so that we have to assume $\beta_{1}>\beta_{2}$ for a consistent solution to exist. In other words, having a family must be more important than child quality expenditure. This parameter restriction is assumed to hold henceforth.

Note that the wish to spend more on children when $\bar{\pi}$ rises cannot be driven by the motive to improve child survival because the starting point of the whole chain of effects was that child health expenditure became less effective with rising $\bar{\pi}$. Thus, it must be driven by the child quality motive. Child expenditure changes its character with improving extrinsic survival probabilities. At low $\bar{\pi}$ 's expenditure is driven by its $\beta_{1}$-component, i.e. health and nutrition, whereas at high $\bar{\pi}$ 's it is mainly motivated by the $\beta_{2}$-component, i.e. schooling and education. The downside of this effect is that it holds also vice versa: parents react to deteriorating child survival by substituting child expenditure with increasing fertility. Thus, with endogenous health, parents in high mortality environments have a comparative advantage in child bearing i.e. in producing cheap children. ${ }^{8}$

\footnotetext{
${ }^{8}$ I borrowed this expression from Moav (2005) who derives a similar result in a very different setting where well educated mothers have a comparative advantage in teaching and poorly educated mothers in child bearing.
} 
From the first order conditions and budget constraint we obtain the solution

$$
\begin{aligned}
c & =\frac{y+\beta_{1} \bar{c}}{1+\beta_{1}}, \\
n & =\frac{(1-\mu \bar{\pi})(1-\bar{c} / y) \beta_{1} \beta_{2} \lambda}{\left(\beta_{1}-\beta_{2}\right)\left(1+\beta_{1}\right) \bar{\pi}}, \\
h & =\frac{\left(\beta_{1}-\beta_{2}\right) \bar{\pi}}{(1-\mu \bar{\pi}) \beta_{2} \lambda} .
\end{aligned}
$$

Taking derivatives confirms that fertility is lower and child expenditure higher under better extrinsic survival conditions.

$$
\begin{aligned}
\frac{\partial n}{\partial \bar{\pi}} & =-\frac{(1-\bar{c} / y) \beta_{1} \beta_{2} \lambda}{\left(\beta_{1}-\beta_{2}\right)\left(1+\beta_{1}\right) \bar{\pi}^{2}}<0 \\
\frac{\partial h}{\partial \bar{\pi}} & =\frac{\beta_{1}-\beta_{2}}{(1-\bar{\mu} \pi)^{2} \beta_{2} \lambda}>0 .
\end{aligned}
$$

Using (6) and (7) we get a simple expression for the survival elasticity of child demand: $(\partial n / \partial \bar{\pi})$. $\bar{\pi} / n=-1 /(1-\mu \bar{\pi})$. Without the health multiplier (i.e. with $\mu=0)$ a one percentage increase in extrinsic child survival would lead to a one percentage decrease of fertility everywhere irrespective of the geo-economic factors determining $\bar{\pi}$. With the health multiplier at work $(\mu>0)$ the elasticity is always larger than one and furthermore increasing in $\bar{\pi}$. Analogously, we obtain the survival elasticity of child expenditure as $(\partial h / \partial \bar{\pi}) \cdot \bar{\pi} / n=1 /(1-\mu \bar{\pi})$. From these elasticities we conclude that ceteris paribus a one percentage increase of the extrinsic survival rate leads to a relatively higher child quantity-quality substitution when $\bar{\pi}$ is already high, i.e. for economies at high geographic latitudes and for economies of high average income.

There is also a positive direct effect of individual income on fertility caused by the incidence of subsistence consumption. As income rises above subsistence level, current consumption becomes less essential and the desire to have children becomes more important for young adults.

$$
\frac{\partial n}{\partial y}=\frac{(1-\bar{\pi}) \beta_{1} \beta_{2} \lambda \bar{c}}{\left(\beta_{1}-\beta_{2}\right)\left(1+\beta_{1}\right) \bar{\pi} y^{2}}>0
$$

The income elasticity of child demand, $(\partial n / \partial y)(y / n)=\bar{c} /(y-\bar{c})$, is infinite at subsistence level and decreases towards zero as income goes to infinity. Later, in the macroeconomic model the survival-effects in (7) are sufficient to explain a delayed demographic transition and slow growth for geographically unfavorable environments. The subsistence-effect in (8), however, is necessary to generate a locally stable poverty trap. 


\section{Correlations of Demo-Economic Development}

Turning towards the macro-economy we impose symmetry, i.e. $\bar{y}=y$. The rate of population growth, $g_{L}:=n(y, \bar{\pi}(y, g)) \cdot \pi(y, g)-1$, is thus affected by income directly through its impact on the fertility decision and, indirectly, through its impact on extrinsic survival probabilities and through this channel on child nutrition and health. The total effect of a marginal increase of income on population growth consists of three parts:

$$
\frac{d g_{L}}{d y}=\underbrace{\frac{\partial n}{\partial y} \cdot \pi}_{\begin{array}{c}
\text { hierarchy of needs } \\
\text { effect }
\end{array}}+\underbrace{\frac{\partial n}{\partial \bar{\pi}} \cdot \frac{\partial \bar{\pi}}{\partial y} \cdot \pi}_{\begin{array}{c}
\text { quantity-quality } \\
\text { substitution effect }
\end{array}}+\underbrace{\frac{\partial \pi}{\partial y} \cdot n}_{\begin{array}{c}
\text { mortality } \\
\text { effect }
\end{array}} .
$$

The first term, the hierarchy-of-needs effect, is positive because parents want to support a larger family as income rises from subsistence level. The second term, the quality-quantity substitution effect, is negative because higher average income increases the probability that children survive and causes parents to substitute fertility with child expenditure. The third term, the mortality effect, is is positive because more children survive when income rises, both because increasing average income in the economy improves the extrinsic survival rate and because parents spend more on each child.

After inserting (2), (6b), (6c), and their respective derivatives into (9) and applying some algebra we get an easily interpretable expression.

$$
\frac{d g_{L}}{d y}=n \pi\left[\frac{\bar{c}}{y(y-\bar{c})}-\left(\frac{1}{(1-\mu \bar{\pi}) \bar{\pi}}-\frac{1}{\bar{\pi}}\right) \frac{\partial \bar{\pi}}{\partial y}\right]=n \pi\left(\frac{\bar{c}}{y(y-\bar{c})}-\frac{\mu}{1-\mu \bar{\pi}} \frac{\partial \bar{\pi}}{\partial y}\right)
$$

It shows that without the health multiplier, i.e. for $\mu=0$, the quantity-quality substitution effect (i.e. the second term) and the mortality effect (the third term) would balance each other. In this case only the hierarchy-of-needs effect (the first term) would remain. This term is infinitely large at subsistence level and vanishes with rising income. The resulting positive correlation of population growth and income would support a globally stable Malthusian equilibrium of stagnation.

With the health multiplier at work, however, the quantity-quality substitution effect is everywhere larger than the mortality effect and furthermore increasing as income rises. The aggregate effect of quantity-quality substitution and mortality on population growth is negative and possibly overcompensating the positive hierarchy-of-needs effect. This will, of course, never be the case when income is close to subsistence and the hierarchy-of-needs effect is close to infinity. 
Figure 3: Patterns of Population Growth

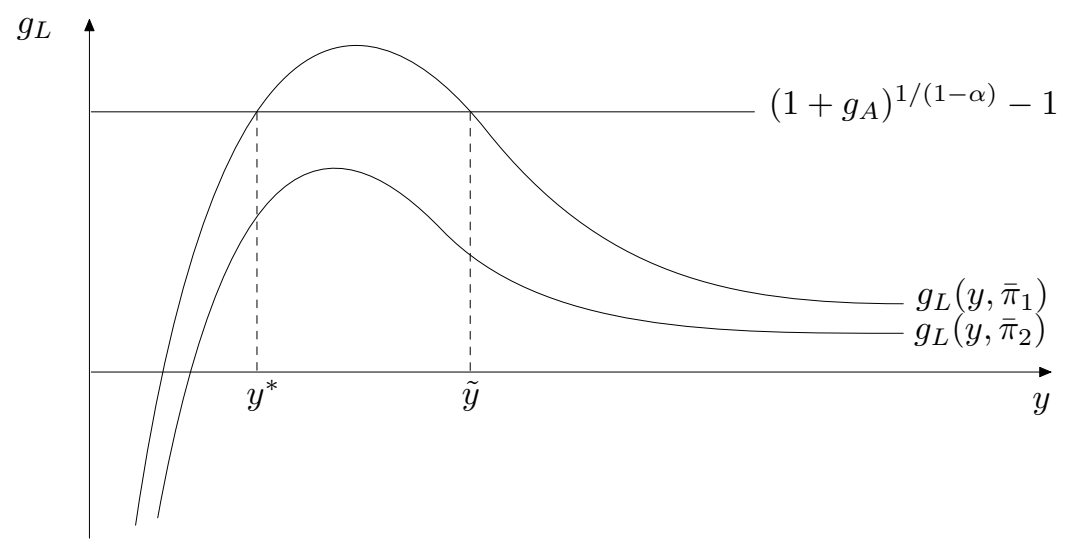

Overcompensating becomes more likely at higher income levels because the hierarchy-of-needs effect dies out very quickly (i.e. quadratically) as income rises. At the income level where all three effects balance each other population growth attains a maximum in an $y-g_{L}$-space. If income rises further the correlation becomes negative. Eventually, the negative effect also vanishes because of the declining impact of average income on extrinsic child survival $\left(\lim _{y \rightarrow \infty} \partial \bar{\pi} / \partial y=0\right)$ and population growth approaches a constant. Summarizing, the model generates a humpshaped correlation between population growth and income.

At the same time the partial effect of extrinsic child survival on population growth is negative for any given income level. Using (6b) and (7a) we obtain ${ }^{9}$

$$
\frac{\partial g_{L}}{\partial \bar{\pi}}=\frac{\partial n}{\partial \bar{\pi}} \cdot \pi+n \cdot \frac{\partial \pi}{\partial \bar{\pi}}=-\frac{n \pi}{(1-\mu \bar{\pi}) \bar{\pi}}+n\left(1-\mu \lambda h+(1-\mu \bar{\pi}) \lambda \frac{\partial h}{\partial \bar{\pi}}\right)=-\frac{n \mu \pi}{1-\mu \bar{\pi}}<0 .
$$

Consider two regions $g_{1}$ and $g_{2}$ with $\bar{\pi}_{1}=\bar{\pi}\left(y, g_{1}\right)<\bar{\pi}_{2}=\bar{\pi}\left(y, g_{2}\right)$. According to (11) the population grows at a higher rate in region 1, the region of lower extrinsic survival probability, for any given level of income. Irrespective of whether the demo-economic system is in its first phase of positive correlation between income and population growth or in its second phase of negative correlation, parents in the unfavorable region always have a comparative advantage in fertility. In other words, at geographically favorable locations with high extrinsic survival rates child expenditure consists to a lesser degree of $\beta_{1}$-components motivated by child survival (i.e. nutrition and health expenditure) and to a higher degree of $\beta_{2}$-components motivated by child

\footnotetext{
${ }^{9}$ Note that there would be no effect of extrinsic child survival on population growth without the health multiplier.
} 
quality (i.e. schooling). Thus, the Beckerian child-quality trade-off is stronger; child expenditure substitutes family size more easily in favorable locations. Figure 3 summarizes the results. The equilibria $y^{*}$ and $\tilde{y}$ will be explained later.

In order to investigate hump-shapednees of population growth and other correlations of demoeconomic development quantitatively, we consider a calibration of the model. For better interpretation generational growth rates are transformed into annual ones. Thus, $y$ is measured as income per year. Let $\psi$ denote the length of adulthood measured by the fecundity period. Annual population growth is then $\gamma_{L} \equiv\left(1+g_{L}\right)^{1 / \psi}-1$. We set $\psi$ to 25 .

Extrinsic child survival is parameterized as $\bar{\pi}=a \cdot\left(1-e^{-b \cdot y}\right)$ so that mortality decays exponentially at rate $b$ when income rises. In other words, survival $\bar{\pi}$ is a concave function of income, reaching a maximum at $a$. The functional form is taken from Kalemli-Ozcan's (2002) empirical work. Yet, we cannot adopt her parameter estimates one-to-one because now $\bar{\pi}$ is only the first of two parts of total child survival. Survival is also determined by individual health expenditure i.e. parameters of the utility function. Therefore $a$ and $b$ are determined in an iterative way together with preference parameters so that the endogenously generated total survival rate corresponds with the actually observed data. This leads to an estimate of $a=0.72$ and $b=0.004$.

Preference parameters are set so that parents in a fully developed country located in temperate climate spend $20 \%$ of their income per child, all children survive childhood, and families consist of 1.2 children per parent (implying a population growth rate of 0.5 percent). These values are chosen to reflect approximately the demo-economic performance of the United States. With the normalization $\mu=1$ they lead to an estimate of $\beta_{1}=0.315$ and $\beta_{2}=0.227$, and $\lambda=5$. Data sources for calibration were USDA (2004) and World Bank (2004).

The subsistence level $\bar{c}$ is set so that population growth at temperate zones peaks at a value of 1.4 percent per year (see Reher, 2004). The resulting income correlations with intrinsic and extrinsic child survival rates, fertility, child expenditure, and population growth are displayed by solid lines in Figure 4 .

Parental behavior generates a positive correlation of income and human capital expenditure and an inverted-u shaped correlation of income and fertility and population growth. The dotted line in the $y-\pi$-diagram represents Kalemli-Ozcan's estimate of the survival function (2002, 
Table 3, survival probability to age 5 in 1997 for 86 countries). One sees that the endogenously generated child survival function approximates the estimate quite well.

Figure 4: Correlations of Demo-Economic Development
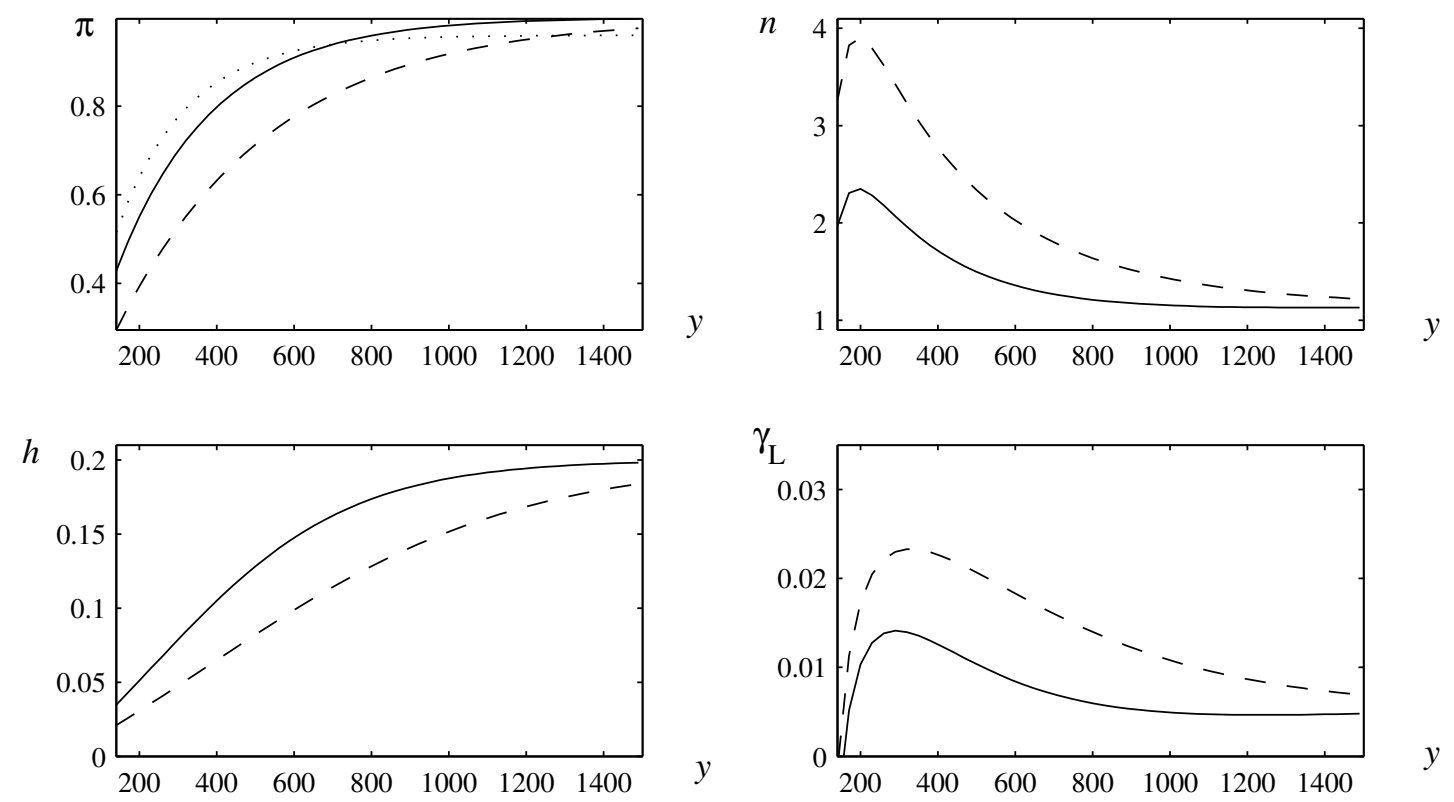

$\beta_{1}=0.315, \beta_{2}=0.227, \mu=1, \lambda=5, \bar{c}=100, a=0.72$, and $b=0.004$ (solid lines) and 0.0025 (dashed lines). Dotted line: estimate of the child survival function in Kalemli-Ozcan (2002, Table $3)$.

Dashed lines in Figure 4 show behavior of families living in an unfavorable environment (a tropical region) where extrinsic child survival improves less quickly when income per capita rises. For that purpose we set $b=0.0025$ and keep everything else from the U.S. calibration. Fertility and population growth peak now at higher levels and decay less quickly. At the peak a couple of adults gives birth to about 8 children generating a population growth rate of 2.4 percent. Note that in the tropical region population growth is not only everywhere higher but also peaks later, i.e. at a higher level of income. Both effects will slow down demo-economic development and increase the possibility of being caught in a poverty trap. A fate that is probably not shared by the otherwise identical country located in a temperate zone. 


\section{Firms And the Macro-Economy}

In order to provide an answer to the question of whether a full demographic transition occurs and how geographic location affects the pace of this process we place the households in a macro model and investigate the dynamic consequences of their actions.

A large number of firms produces with a constant returns to scale technology using labor and land, $X$. Labor supply is given by the number of young adults, $L$, times their human capital endowment, $\tilde{h}$. Supply of (arable) land is fixed, technological progress is exogenous, and the production function is of Cobb-Douglas type.

$$
Y_{t}=A_{t}\left(L_{t} \tilde{h}_{t}\right)^{\alpha} X^{1-\alpha}, \quad 0<\alpha \leq 1 .
$$

Labor is paid according to its marginal product so that after normalizing land supply to one income per adult is obtained as

$$
y_{t}=\alpha A_{t} L_{t}^{\alpha-1} \tilde{h}_{t}^{\alpha}
$$

By focussing on labor income we ignore fertility behavior of landlords. ${ }^{10}$

Let the human capital endowment of the current work force be determined by child expenditure of their parents, $\tilde{h}_{t+1}=f\left(h_{t}\right)$. Because the number of workers depends on fertility of their parents (and child survival rates at that time), and both fertility and child expenditure depend on income, equation (13) provides the link between generational income levels. Next generation's income is given by

$$
y_{t+1}=\alpha\left(1+g_{A}\right) A_{t}\left[\left(1+g_{L}\left(y_{t}\right)\right) \cdot L_{t}\right]^{\alpha-1} \cdot\left[f\left(h\left(\bar{\pi}\left(y_{t}\right)\right)\right)\right]^{\alpha} .
$$

Justified by the focus of analysis on developing economies we assume that productivity grows at an exogenously given rate $g_{A}, g_{A}:=\left(A_{t+1}-A_{t}\right) / A_{t}$ for all $t, t+1$. Let $x_{t} \equiv A_{t} / L_{t}^{1-\alpha}$ denote a labor supply adjusted measure of productivity and let us for notational convenience assume that human capital expenditure of parents relates one-to-one to human capital endowment of

$\overline{{ }^{10} \text { Alternatively }}$ and without impact on qualitative results we could have assumed family-managed firms and labor paid according to its average product. See Strulik (2005) for a longer version that integrates physical capital. 
their children, $\tilde{h}_{t+1}=h_{t}$. Macroeconomic dynamics are then determined by the following twodimensional system. ${ }^{11}$

$$
\begin{aligned}
& y_{t+1}=\alpha\left(1+g_{A}\right)\left[1+g_{L}\left(y_{t}\right)\right]^{\alpha-1} h\left(y_{t}\right)^{\alpha} x_{t}, \\
& x_{t+1}=\left(1+g_{A}\right)\left(1+g_{L}\left(y_{t}\right)\right)^{\alpha-1} x_{t} .
\end{aligned}
$$

Note that both $x_{t}$ and $y_{t}$ are predetermined. Adjustment dynamics will thus be unique.

\section{Stagnation}

From (14) follows that any equilibrium fulfils

$$
g_{L}(y)=g_{L}^{*} \equiv\left(1+g_{A}\right)^{1 /(1-\alpha)}-1
$$

At an equilibrium, the positive impact of technological progress is neutralized by the negative impact of population growth (through decreasing returns to scale with respect to the reproducible factors). For existence of this Malthusian-like equilibrium the fertility rate supporting $g_{L}{ }^{*}$ has to lie in the feasible range of parental preferences, i.e. $g_{L}{ }^{*} \leq \max g_{L}(y)$. Inspection of (15) shows that existence of an equilibrium becomes increasingly unlikely for any set of preference parameters when technological progress grows faster or when arable land becomes a less essential factor in production (i.e. $\alpha$ rises).

Consider, for example, an economy populated by parents with preferences as those underlying the income correlations in Figure 4 (dotted lines). These parents generate a maximum population growth rate of 2.4 percent annually implying that an equilibrium exists if $\left(1+g_{A}\right)^{1 /(1-\alpha)}-1 \leq$ 0.024. For example, if technological progress grows at 0.5 percent p.a., an equilibrium exists for $\alpha \leq 0.79$. If $g_{A}$ is rises to one percent per year, existence requires $\alpha<0.58$. For given geographic location, economies particularly susceptible for stagnation are characterized by low agricultural productivity growth and high dependency on arable land.

Yet, economies are actually located at different latitudes and the analysis in Section 3 has shown (Figures 3 and 4) that population growth is higher at unfavorable geographic locations. This implies that for given parameters of preference and technology, existence of equilibrium is

\footnotetext{
${ }^{11}$ Because $h$ is bounded from above and below through household behavior, subsequent results are robust for any positive function $f(h)$. Furthermore, we could introduce technological progress as being determined by the skill level of the working population - as in Galor and Weil (2000) and Strulik (2004b) - without change in qualitative results.
} 
more likely for unfavorably located economies. For example, with $g_{A}=0.5$ percent and $\alpha=0.79$ the equilibrium does not exist for the U.S. calibration of Figure 4 (solid lines) while it exists for the tropically located country (dashed lines).

If an equilibrium exists, it may be unstable. Stability requires that a "Malthusian mechanism" operates according to which $\partial g_{L} / \partial y>0$ implying that there is at most one stable equilibrium. In order to prove this claim we exclude the degenerate case where $g_{L}^{*}$ is exactly at $\max \left(g_{L}\right)$. Then, the hump-shaped curvature of the $g_{L}(y)$ curve ensures that either none or two equilibria exist, and - if two equilibria exist - that we observe $\partial g_{L} / \partial y>0$ at the first one (labelled $y^{*}$ ) and $\partial g_{L} / \partial y<0$ at the second one (labelled $\tilde{y}$ ), see Figure 3.

The elements of the Jacobian matrix $J$ of system (14) evaluated at an equilibrium are

$$
\begin{aligned}
\frac{\partial y_{t+1}}{\partial y_{t}} & =\alpha x h^{\alpha}\left((\alpha-1)\left(1+g_{L}\right)^{-1} \frac{\partial g_{L}}{\partial y}+\frac{\alpha}{h} \frac{\partial h}{\partial y}\right) \equiv J_{1} \\
\frac{\partial y_{t+1}}{\partial x_{t}} & =\alpha h^{\alpha} \equiv J_{2}>0 \\
\frac{\partial x_{t+1}}{\partial y_{t}} & =-(1-\alpha) x\left(1+g_{L}\right)^{-1} \frac{\partial g_{L}}{\partial y} \equiv J_{3} \\
\frac{\partial x_{t+1}}{\partial x_{t}} & =1 .
\end{aligned}
$$

Local stability requires that both eigenvalues are smaller than one in absolute terms which translates into the condition $\left|1+J_{1}\right|<\left(1+J_{1}\right)-J_{2} J_{3}$. Because $J_{2}>0$, this necessarily requires $J_{3}<0$ i.e. $\partial g_{L} / \partial y>0$. Thus, the equilibrium at $\tilde{y}$ is never stable.

Only the equilibrium at $y^{*}$ - where the Malthusian mechanism operates - is a potential candidate for a stable poverty trap. Substituting (14a) evaluated at the equilibrium into (16) we observe that the condition $\partial g_{L} / \partial y>0$ becomes sufficient together with

$$
1+\alpha \frac{\partial h}{\partial y} \frac{y}{h}>\frac{1}{2}(1-\alpha) \frac{\partial\left(1+g_{L}\right)}{\partial y} \frac{y}{1+g_{L}} .
$$

Condition (17) requires that the income elasticity of population growth is not too large. If it is too large, the equilibrium becomes unstable and low-income economies converge towards subsistence level. It can be verified numerically that (17) is not restrictive and $y^{*}$ is - if it exists - stable for any reasonable parameterization of the model. Note, however, that $y^{*}$ is only locally stable. Any big push or series of small positive shocks that drives $y$ in regions where 
$\partial g_{L} / \partial y<0$ enables an escape towards successful demo-economic development. The following theorem summarizes the results.

THEOREM 1. There exists a non-empty set of preference and technology parameters for which a locally stable equilibrium of low income and high population growth exists in a geographically unfavorable environment but not in a geographically favorable environment.

Example: The calibration for the U.S. shown in Figure 4 and $g_{A}=1 / 2$ percent and $\alpha=0.8$ renders the equilibrium non-existent. Keeping U.S. parameters of preferences and technology the equilibrium exists for location parameters $b \leq 0.0024$.

\section{The Pace of Demo-Economic Transition}

The model offers an explanation for the observation that the pace of demographic transition is influenced by geographic location. In particular at tropical locations the pattern of demographic transition can look very differently compared to the experiences of the Western world. In fact, focussing on population growth alone, ongoing demographic transition can be so exceedingly slow that it looks like stagnation within a reasonable time-window (of, say, 50 years). Because it is visually indistinguishable, the situation may mistakenly be identified as poverty trap.

In order to demonstrate these results, we consider the following experiment. Suppose there are three countries sharing the parameters of preference for consumption, family size, and child expenditure with the U.S. calibration from Figure 4. Assume identical technologies for all three countries: $\alpha=0.8$ and $g_{A}=0.04$ percent. Given the low rate of technological progress, the population grows initially at a low equilibrium rate of 0.2 percent everywhere. The three countries differ in their extrinsic child survival rates, parameterized by $b \in\{0.004,0.0025,0.002\}$.

For purpose of comparison we normalize time so that all countries share a common onset of the demo-economic transition. At time $t=0$ the countries experience a permanent shock of productivity growth towards $g_{A}=0.5$ percent. The new steady state of stagnation would be at $g_{L}^{*}=2.5$ percent. It exists for the tropically located country where $b=0.002$ but does not exists for the other two countries, which both undergo a demographic transition with extrinsic child survival converging towards $\bar{\pi}=a$. Eventually they will display the same fertility rate, child expenditure, and income growth. Their ways taken towards this balanced growth path, however, are different. 
Consider first the geographically favorably located country where $b=0.004$. Time paths (impulse response functions) induced by the increase of $g_{A}$ are represented by solid lines in Figure 5. Driven by technological progress and human capital accumulation the economy expands in the first phase at relatively high rates of income growth $\left(\gamma_{y}\right)$ above 2 percent. Because of the hierarchy-of-needs effect parents want to have larger families initially and population growth rises. Yet, this effect is comparatively small and of short duration. After about 35 years survival probabilities are sufficiently good so that the effect of quality-quantity substitution dominates the hierarchy-of-needs effect and the mortality effect and population growth begins to decline. After about 150 years the demo-economic transition comes to its end and the system stabilizes along a balanced growth path.

Figure 5: Patterns of Development
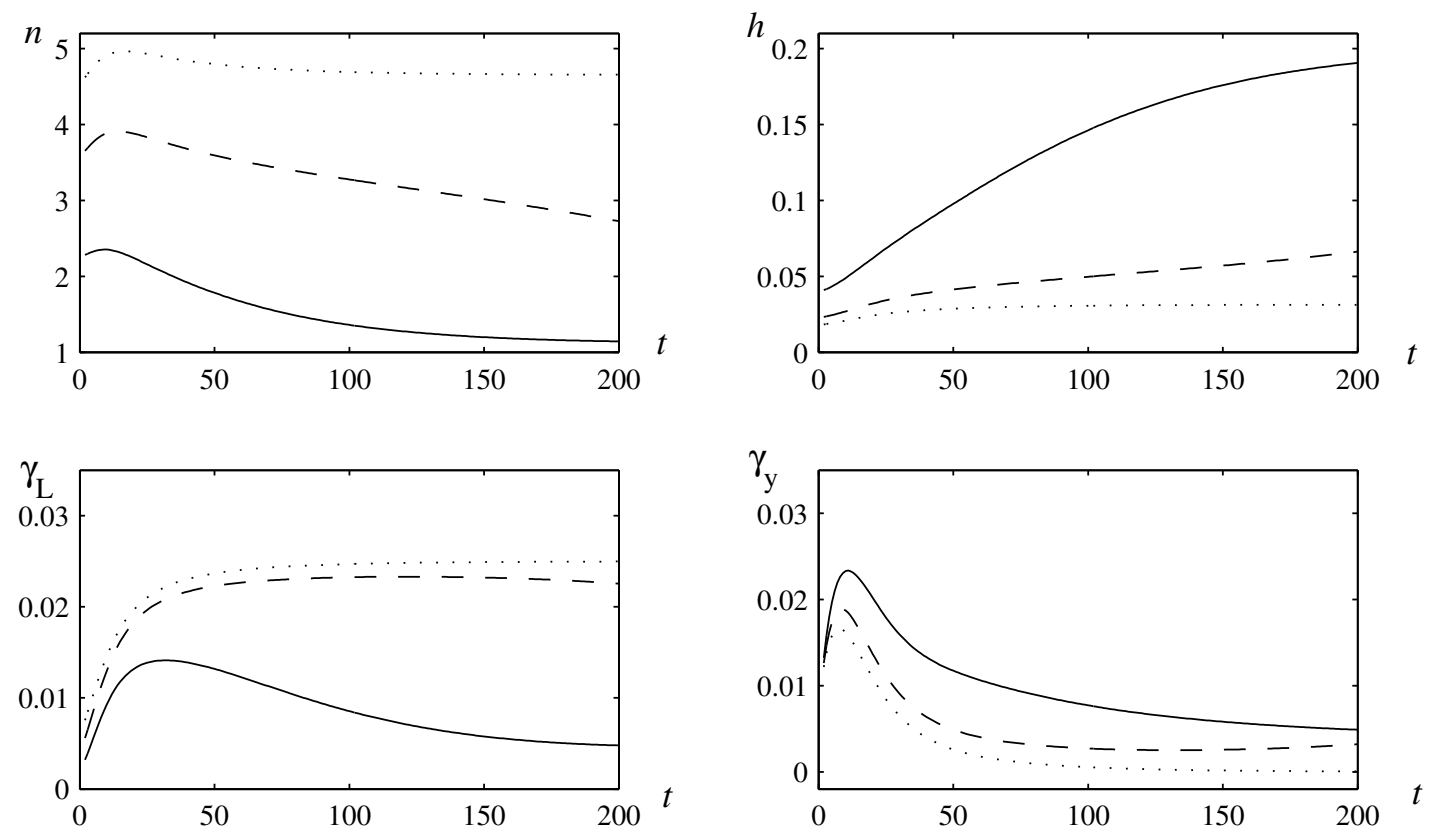

Parameters as for Figure 4 and $g_{A}=0.005$, and $\alpha=0.8$. Solid lines: $b=0.004$, dashed lines: $b=0.0025$, dotted lines: $b=0.002$.

Now consider the transition of the unfavorably located country where $b=0.0025$. Time paths are represented by dashed lines. This country begins also to grow economically after the positive technology shock but at a lower rate than the favorably located one. Potential positive income effects are more heavily absorbed by population growth. After a short period of promising development economic growth begins to decline towards almost zero. At this low 
level of economic growth fertility declines much more gradually and its impact on population growth is almost compensated by improvements of child survival. As a consequence population growth remains almost constant at a high level. Observers (of only the time window time displayed in Figure 5) may think that the country has stabilized at a low-level poverty trap. In fact, the economy develops, albeit exceedingly slow. We can prove analytically that there exist no steady-state of stagnation, so that the demo-economic system will reach its turning point where population growth begins to decline and the second phase of transition ignites so that the economy converges towards the U.S. growth path eventually.

Finally, consider an even worse scenario where $b=0.002$, represented by dotted lines. From our analytical results we know that now the strong reaction of population growth causes stagnation at the poverty trap. The resulting time path of population growth, however, almost coincides with the path of the economy represented by dashed lines, which eventually manages the demographic transition. Given the relatively short time series of actual data, the results suggest that it could be hard to tell stagnation from ongoing development at exceedingly slow speed. It may also shed new light on the modelling of unsuccessful development as locally stable equilibrium of stagnation.

Let us now assume that all countries of the world eventually manage a demographic transition. An alternative way to generate this scenario within the present model (besides, assuming parameters that do not support a poverty trap) would be to consider stochastic technological progress. A demographic transition is successfully initiated if population growth moves out of the domain of attraction of the locally stable equilibrium at $y^{*}$. Thus, it takes a small series of positively correlated technology shocks to move population growth sufficiently "over the hump" in geographically favorably located regions, whereas it takes a long series of positive shocks (or a big push) to arrive at similar results in unfavorably located regions. In other words, given the same stochastic process for technology it is more likely at high geographic latitudes that a successful demographic transition occurs early.

In any case, irrespective of whether we focus on geographic's impact on duration only or additionally on its onset, the observed patterns of population growth implies that we observe economic divergence from cross-country perspective during the transitional phase. This relates the present paper to Lucas (2002) who argues that ultimately "we will see a world that, economically, looks more and more like the United States today" but that different speeds of transition 
are observed based on the heterogeneity of human capital accumulation and the child qualityquantity trade-off. Here we have presented a theory that explains who are the leaders and laggers in this process i.e. why it is no coincidence that tropically located countries are lagging behind.

\section{Related Literature}

According to Barro and Becker's (1988) macroeconomic model of optimal fertility choice and child quality-quantity trade-off (and the literature based thereon) higher child survival rates should cause population growth not be lower and possibly higher. In other words, population growth should not peak at lower and possibly at higher rates in geographically favorably located countries. So far the literature has produced two ways to reconcile theory with the observed facts. One way - followed by most authors - was to assume that the fertility transition evolved independently from the mortality transition or was at least not crucially determined by changes of mortality. The other way was the theory of precautionary child bearing.

The theory of precautionary child bearing (Sah, 1991, Kalemli-Ozcan, 2002, 2003) investigates the impact of uncertainty about the number of surviving children on fertility and population growth. The curvature of the utility function - reflecting risk aversion of the stochastically optimizing parent - determines whether parents react on improving child survival with higher or lower fertility. A crucial assumption besides sufficiently strong risk aversion is that there exists a unique date of a discrete fertility decision. If one considers sequential child bearing so that parents are able to replace children who die early, then the positive correlation between child mortality and population growth disappears. This has recently been shown by Doepke (2005) who therefore argues in favor of the easier to handle deterministic model because it "leads to virtually the same conclusions as the stochastic model with sequential fertility choice."

Some of the ideas developed in the present paper were already apparent in Blackburn and Cipriani (1998) and Strulik (2004b). Blackburn and Cipriani investigate a Barro-Becker (1988)type model with endogenous health and mortality and focus on transitional dynamics that are consistent with the historic successful development of the Western world, while the present paper investigates the impact of geography on delayed and possibly stalled demographic transition in today's least developed countries. In Strulik (2004b) the central message was obscured by some non-standard assumptions about the utility function leading to an awkward differentiation 
between interior and corner solutions. Here, the paper has introduced a proper distinction between intrinsic and extrinsic survival rates and has employed the incidence of subsistence consumption to derived its central results more stringently without recurring on corner solutions. The impact of geography on the pace of demographic transition was not investigated in the earlier articles. The present paper shares also some basic ideas with Galor and Moav (2005). The main difference is that Galor and Moav focus on an evolutionary explanation of intrinsic mortality whereas the present paper explains intrinsic mortality as individually controlled through child expenditure. $^{12}$

The present paper is also related to articles investigating the influence of longevity on human capital accumulation and economic growth. ${ }^{13}$ Sharing some of the demo-economic mechanisms at work, the present paper deviates from this literature by its focus on child mortality, geography, and nutrition. Ehrlich and Lui (1992) and Soares (2005) investigate fertility and educational choice when both adult and child survival are uncertain. Lagerlöf (2003) presents a model of the onset of the demographic transition in the Western world. Endogenous fertility under extrinsic mortality and subsistence consumption is investigated by Jones (2001). Strulik (2004a) proposes a link of fertility, mortality, and child labor. Corrigan et al. (2005) investigate the influence of the AIDS epidemic on human capital accumulation and growth through the creation of orphans. The main difference to these articles is the decomposition of extrinsic and intrinsic survival rates, and the role of geography, nutrition, and health expenditure in the present paper.

It is also interesting to contrast the current paper with Galor and Weil's "unified growth theory" (Galor and Weil, 2000, Galor and Moav, 2002, Galor, 2005). The common basic idea is that a permanent escape from poverty cannot be explained without a demographic transition and vice versa. The main difference results from the consideration of mortality. In unified growth theory declining fertility is assumed to be triggered by human capital accumulation. The majority of papers in this spirit consequentially neglect mortality and focus on the interplay of a "fertility transition" with economic development. It is argued that the historical pattern of a fertility reversal, i.e. first increasing and then decreasing fertility during a period in which mortality is continuously falling is not compatible with the (standard) theory of demographic

\footnotetext{
${ }^{12}$ Recently, Galor et al., 2006, proposed a theory that explains how geographic location shaped the pace of transition from agriculture to manufacturing. There, geography, operates through a different channel namely land inequality, institution building, and the accumulation of human capital. Mortality, fertility, and demographic transition are not considered.

${ }^{13}$ See, e.g. Blackburn and Cipriani (2002), Boucekkine et al. (2003), Chakraborty (2004), and Cervelatti and Sunde (2005). See Hazan and Zoabi (2006) for a critically discussion of longevity's influence on economic growth.
} 
transition according to which decreasing mortality causes decreasing fertility. Yet, here we take from demography the idea that the fertility transition cannot be explained without mortality change (see Lee, 2003) and explain how nevertheless the resulting path of development can encompass a fertility reversal, i.e. first rising and then decreasing birth rates while mortality is continuously falling. ${ }^{14}$

\section{FinAl REMARKS}

This article has offered a theory that explains why population growth is high at geographically unfavorable (tropical) locations and how this may cause a particularly poor demo-economic performance of these regions. Thereby it has become obvious that the question whether a country is stuck in a population- or poverty-trap or whether no such thing exists may be of second-order practical relevance. ${ }^{15}$ For an unfavorably located region an ongoing demographic transition can be so exceedingly slow that it becomes indistinguishable from actual stagnation within a reasonable time frame.

It may be helpful to summarize the mechanics of the model by explaining what would happen if - successively - one of its central elements were absent. For example, if we would replace the Malthusian assumption of decreasing returns in production by constant returns (i.e. if we neglect the importance of land), then there would be no poverty trap. Population growth would no longer affect the pace of development directly. Nevertheless, the theory would continue to predict that population growth is higher and economic development is slower at unfavorable latitudes. Slower economic development would then be based solely on the slower growth of human capital at low latitudes (see Figure 4).

If we would neglect subsistence consumption, there would be no non-linear adjustment of fertility behavior. From this follows that there would be no hump-shaped population growth (because the quantity-quality substitution effect overcompensates the mortality effect) and thus no poverty trap. Nevertheless countries at low latitudes would grow slower economically because population growth is higher, the demographic transition is slower, and human capital expenditure is lower.

\footnotetext{
${ }^{14} \mathrm{~A}$ further difference with unified growth theory is that we make no effort in explaining the interplay of demographic development and technological progress. This simplifying assumption is justified by the focus on today's less developed countries. The possibility of endogenous technological progress could be added at the expense of more analytical complexity without changing the main insights the paper wants to convey.

${ }^{15}$ Conceding, of course, that it can be an interesting theoretical issue. See Bloom et al. (2003) for a special investigation on geographic determinism versus poverty traps.
} 
Finally, if we would drop the health multiplier, mortality effects and quantity-quality substitution effects would balance each other. Thus, given subsistence consumption the poverty trap would be globally stable. If we would additionally abandon subsistence needs there would be no correlation between income and population growth anymore.

The proposed economic channel based on child survival and health costs complements other, socio-cultural explanations of high population growth in Sub-Saharan Africa and other tropical regions (e.g. Chesnais, 1992). The main advantage compared to explanations built on differences of preferences is that policy recommendations are straightforward to be drawn from the cost channel. In short, a health policy accomplishing that all diamonds in Figure 1.a are located on a horizontal line at survival rates of today's fully developed economies disables the proposed mechanism for slow or stagnant economic development. Thus, geography's indirect impact on development could, at least theoretically, be overcome by improvements of nutrition and by disease eradication. This slightly optimistic outlook distinguishes the theory from the institutions-based approach where the indirect influence of geography originates from the now unchangeable mortality rates about 200 years ago.

Technological progress has deliberately been modelled as being exogenous because the paper does not claim to contribute to the new field of "unified growth theories" that explains development of countries at the technological frontier (see Galor, 2005). Conversely, comparing countries at different latitudes, it has been shown that there may exist non-unifiable patterns of development. In Pritchett's (2000) words the theory helps to explain why time paths of demoeconomic variables now display plateaus and valleys where there were only hills visible at times of the Western world's demographic transition.

There are extensions of the model available. In Strulik (2005) the model is extended by a third period and explained why the savings rate increases with demo-economic development. Strulik (2007) provides an extension towards a two-sector model that explores the interaction of demographic transition and structural change, i.e. how geography shapes the pace of industrialization. Ongoing research tries to integrate a deeper explanation of the interaction of subsistence needs and human health using a biological foundation of ontogenetic growth (Dalgaard and Strulik, 2006). 


\section{References}

Acemoglu D., Johnson S., Robinson J.A., 2001, The Colonial Origins of Comparative Development: An Empirical Investigation, American Economic Review, 91 (5), 1369-1401.

Andreoni, J., 1989, Giving with Impure Altruism: Applications to Charity and Ricardian Equivalence, Journal of Political Economy 97(6), 1447-1458.

Barro, R.J., and G.S. Becker, 1988, A Reformulation of the Economic Theory of Fertility, Quarterly Journal of Economics 57, 481-501.

Becker, G.S., 1960, An Economic Analysis of Fertility. In: National Bureau of Economic Research (ed), Demographic and Economic Change in Developed Countries, Princeton University Press, Princeton, 209-231.

Black, R.E., S.S. Morris, and J. Bryce, 2003, Where and Why are 10 Million Children Dying Every Year?, Lancet 361, 2226-2234.

Blackburn, K., and G.P. Cipriani, 1998, Endogenous Fertility, Mortality and Growth, Journal of Population Economics 11, 517-534.

Blackburn, K., and G.P. Cipriani, 2002, A Model of Longevity, Fertility and Growth, Journal of Economic Dynamics and Control 26, 187-204.

Bloom, D.E. and J.D. Sachs, 1998, Geography, Demography, and Economic Growth in Africa, Brookings Papers on Economic Activity, 1998:2, 207-295.

Bloom, D.E., D. Canning, and J. Sevilla, 2003, Geography and Poverty Traps, Journal of Economic Growth 8, 355-378.

Boucekkine, R., D. de la Croix, and O. Licandro, 2003, Early Mortality Declines at the Dawn of Modern Growth, Scandinavian Journal of Economics 105, 401-418

Brown, J.H., 1995, Macroecology, Chicago: University of Chicago Press.

Caulfield, L.E., M. de Onis, M. Bloessner, and R.E. Black, 2004a, Undernutrition as an Underlying Cause of Child Deaths Associated with Diarrhea, Pneumonia, Malaria, and Measles, American Journal of Clinical Nutrion 80, 193-198.

Caulfield, L.E., S.A. Richard, and R.E. Black, 2004b, Undernutrition as an Underlying Cause of Malaria Morbidity and Mortality in Children Less Than Five Years Old, American Journal of Tropical Medicine and Hygiene 71, 55-63.

Cervelatti, M. and U. Sunde, 2005, Human Capital Formation, Life Expectancy and the Process of Economic Development, American Economic Review 95, 1653-1672.

Chakraborty, S., 2004, Endogenous Lifetime and Economic Growth, Journal of Economic Theory $116,119-137$.

Chesnais, J.-C., 1992, The Demographic Transition: Stages, Patterns, and Economic Implications, Oxford: Clarendon Press.

Corrigan, P., G. Glomm, and F. Mendez, 2005, AIDS Crisis and Growth, Journal of Devlopment Economics 77, 107-124.

Cutler, D., A. Deaton, and A. Lleras-Muney, 2006, The Determinants of Mortality, Journal of Economic Perspectives 20(3), 97-120.

Dalgaard, C.-J. and H. Strulik, 2006, Subsistence - A Biological Foundation of the Malthusian Equilibriium, Working Paper, Copenhagen University. 
Dasgupta, P., 1993, An Inquiry into Well-being and Destitution, Oxford University Press, Oxford.

Doepke, M., 2005, Child Mortality and Fertility Decline: Does the Barro-Becker Model Fit the Facts?, Journal of Population Economics 18, 337-366.

Ehrlich I, and F.T. Lui, 1991, Intergenerational Trade, Longevity, and Economic Growth, Journal of Political Economy 99, 1029-1059.

Filmer, D. and L. Pritchett, 1999, The Impact of Public Spending on Health: Does Money Matter?, Social Science and Medicine 49, 1309-1323.

Fogel, R.W., 1994, Economic Growth, Population Theory and Physiology: The Bearing of LongTerm Processes on the Making of Economic Policy, American Economic Review 84, 369-395.

Fogel, R.W., 2004, The Escape from Hunger and Premature Death, 1700-2100, Cambridge University Press, Cambridge.

Galor, O. and D.N. Weil, 2000, Population, Technology, and Growth: From Malthusian Stagnation to the Demographic Transition and Beyond, American Economic Review 90, pp. 806-828.

Galor, O., 2005, From Stagnation to Growth: Unified Growth Theory, in: Handbook of Economic Growth, Amsterdam: North-Holland.

Galor, O. and O. Moav, 2002, Natural Selection and the Origin of Economic Growth, Quarterly Journa of Economics 117, 1133-1191.

Galor, O. and O. Moav, 2005, Natural Selection and the Evolution of Life Expectancy, CEPR Discussion Paper No. 5373.

Galor, O., O. Moav, and D. Vollrath, 2006, Inequality in Land Ownership, the Emergence of Human Capital Promoting Institutions, and the Great Divergence, Working Paper.

Guegan, J.F., F. Thomas, M.E. Hochberg, T. de Meeus, F. Renaud, 2001, Disease Diversity and Human Fertility, Evolution 55, 1308-1314.

Harris, B., 2004, Public Health, Nutrition, and the Decline of Mortality: The McKeown Thesis Revisited, Social History of Medicine 17, 379-407.

Hazan, M. and H. Zoabi, 2006, Does Longevity Cause Growth? Journal of Economic Growth $11,363-376$.

Jones, C.I., 2001, Was an Industrial Revolution Inevitable? Economic Growth over the Very Long Run, Advances in Macroeconomics 1(2), Article 1.

Kalemli-Ozcan, S., 2002, Does the Mortality Decline Promote Economic Growth, Journal of Economic Growth 7, 411-439.

Kalemli-Ozcan, S., 2003, A Stochastic model of mortality, fertility, and human capital investment, Journal of Development Economics 70, 103-118.

Kirkwood, T.B. and S.N. Austad, 2000, Why Do We Age?, Nature 408, 233-238.

Lagerlöf, N.-P., 2003, Mortality and Early Growth in England, France and Sweden, Scandinavian Journal of Economics 105, 419-439.

Lee, R., 2003, The Demographic Transition: Three Centuries of Fundamental Change, Journal of Economic Perspectives 17, 167-190.

Lucas, R.E. Jr., 2002, The Industrial Revolution: Past and Future, in: Lucas, R. E. Jr., Lectures on Economic Growth, Cambridge, Massachusetts: Harvard University Press.

Masters W.A., and M.S. McMillan, 2001, Climate and Scale in Economic Growth, Journal of Economic Growth 6, 167-186. 
McKeown, T., 1976, The Modern Rise of Population, Academic Press, New York.

Moav, O., 2005, Cheap Children and the Persistence of Poverty, Economic Journal 115, 88-110.

Olsson, O., and D.A. Hibbs, 2005, Biogeography and Long-Run Economic Development, European Economic Review, forthcoming.

Pelletier, D.L., E.A. Frongillo, and J.P. Habicht, 2003, Epidemiologic Evidence for a Potentiating Effect of Malnutrition on Child Mortality, American Journal of Public Health 83, 1130-1133.

Pritchett L., 2000, Understanding Patterns of Economic Growth: Searching for Hills, among Plateaus, Mountains, and Plains, World Bank Economic Review 14, 221- 250.

Pritchett, L., and L.H. Summers, 1996, Wealthier is Healthier, Journal of Human Resources $31(4), 841-868$.

Reher, D.S., 2004, The Demographic Transition Revisited as a Global Process, Population, Space and Place 10, 19-41.

Rice, A.L., L. Sacco, A. Hyder, R.E. Black, 2000, Malnutrition as an Underlying Cause of Childhood Deaths Associated with Infectious Diseases in Developing Countries, Bulletin of the World Health Organization 78, 1207-1221.

Sachs, J.D., 2003, Institutions Don't Rule: Direct Effects of Geography on per Capital Income. NBER Working Paper 9490.

Sah, R.K., 1991, The Effects of Child Mortality on Fertility Choice and Parental Welfare, Journal of Political Economy 99, 582-606.

Schultz, T.P., 1999, Health and Schooling Investments in Africa, Journal of Economic Perspectives $13,67-88$.

Soares, R.R., 2005, Mortality Reductions, Educational Attainment, and Fertility Choice, American Economic Review 95, 580-601.

Strulik, H., 2004a, Child Labor, Child Mortality, and Economic Development, Economic Journal, 2004, Vol. 114, 547-568.

Strulik, H., 2004b, Economic Growth and Stagnation with Endogenous Health and Fertility, Journal of Population Economics 17, 433-453.

Strulik, H., 2005, Geography, Health, and Demo-Economic Development, Copenhagen University, Discussion Paper 05-15.

Strulik, H., 2007, Demo-Economic Development and the Pace of Industrialization, Hannover University, Discussion Paper.

USDA, 2004, Expenditures on Children by Families, 2003 Annual Report. Washington: U.S. Department of Agriculture.

UNICEF, 2006, Global Database on Child Malnutrition, http://www.childinfo.org/

Williams, G.C., 1957, Pleiotropy, Natural Selection, and the Evolution of Senescence, Evolution $11,398-411$.

World Bank, 2004, World Development Indicators. Washington: World Bank.

World Health Organization, 2002, World Health Report 2002: Reducing Risk, Promoting Healthy Life, Geneva. 